Ашабоков Б.А., 2,1 Высокогорный геофизический институт, Россия Ташилова А.A. ${ }^{1}, \quad 2$ Институт информатики и проблем регионального управления Кешева Л.А. ${ }^{* *}$ КБНЦ РАН, Россия

* kesheva.lara@yandex.ru

\title{
РЕЗУЛЬТАТЫ ПРОГНОЗА СНЕЖНОГО ПОКРОВА В КАВКАЗСКОМ РЕГИОНЕ МЕТОДОМ СИНГУЛЯРНО- СПЕКТРАЛЬНОГО АНАЛИЗА
}

Введение: Изменения режима зимних осадков и снежного покрова могут считаться комплексным индикатором климата холодного сезона, отражающим изменения режима температуры, режима осадков, частоты оттепелей и т.д. Запасы воды в снеге играют определяющую роль при весеннем половодье, влияют на увлажнение почвы при севе яровых и росте озимых культур. Следует подчеркнуть важность знания закономерностей распределения осадков в холодный период для оценки агроклиматических ресурсов

Материалы и методы республики, к которым относится и снежный покров.

исследований

Результаты исследований

Прогноз изменений характеристик снежного покрова является не менее важной проблемой, чем прогноз изменений климата (температуры и жидких осадков). В данной работе на основе данных метеопараметров, предоставленных Северо-Кавказским УГМС, были получены осредненные ряды среднедекадной высоты снежного покрова и числа дней со снежным покровом для юга европейской территории России (ЕTP). С использованием метода сингулярно-спектрального анализа («Гусеница»-SSA) проанализирована динамика и исследованы прогностические возможности метода SSA для высоты снежного покрова и числа дней со снежным покровом на юге ETP. Метод SSA представляет собой инструмент для анализа и прогнозирования одномерных и многомерных временных рядов. На основе проведенного Т-теста показана эффективность применения рекуррентного R-SSA прогноза среднегодовой высоты снега и числа дней со снежным покровом.

и их обсуждение: Для всех рассмотренных метеорологических величин была получена периодичность их изменения, стандартное отклонение, максимальное отклонение и относительная ошибка. В результате подбора главных компонент (1, 3 и 13) были получены прогнозные тенденции изменения исследуемых переменных, выявлены периоды их увеличения и снижения, а также получены прогнозные значения среднедекадной высоты снежного покрова и числа дней для периода 2018-2022 гг.

Выводы: В результате применения метода сингулярно-спектрального анализа осуществлен прогноз таких характеристик снежного покрова юга ЕTP как среднедекадная высота снежного покрова и число дней со снежным покровом юга ETP на 2018-2022 гг. Выявленные общие тенденции изменения исследуемых характеристик снежного покрова юга ЕTP на период до 2022 г. позволяют характеризовать региональные изменения климата юга европейской части России как составную часть современного глобального потепления.

Ключевые слова: высота снежного покрова, сингулярно-спектральный анализ, прогноз, главные компоненты, остатки модели, Т-тест. 
High Mountain Geophysical Institute, Russia

Institute of Informatics and Regional Management Problems, KBNC RAS, Russia
Ashabokov B A.

Tashilova A.A.

Kesheva L.A.

Introduction:

Materials and methods of research:

Results of the study and their discussion:

Conclusions:

Key words:

\section{RESULTS OF SNOW COVER FORECAST IN THE CAUCASUS REGION USING THE METHOD OF SINGULAR-SPECTRAL ANALYSIS}

Changes in the mode of winter precipitation and snow cover can be considered as a complex indicator of the climate of the cold season, reflecting changes in the temperature regime, precipitation mode, the frequency of thaws, etc. Water reserves in the snow play a decisive role during the spring flood, affect soil moisture during the sowing of spring crops and the growth of winter crops. The importance of knowing the patterns of distribution of precipitation during the cold period for assessing the agro-climatic resources of the republic, which includes snow cover should be emphasized.

The forecast of changes in snow cover characteristics is no less important than the forecast of climate changes (temperature and liquid precipitation). In this work, based on the meteorological data provided by the North Caucasian UGMS, we obtained the averaged series of the average decade height of snow cover and the number of days with snow cover for the south of the European territory of Russia (ETR). Using the method of singular-spectral analysis ("Caterpillar" -SSA), the dynamics were analyzed and the prognostic capabilities of the SSA method for the height of snow cover and the number of days with snow cover in the south of ETR were investigated. The SSA method is a tool for analyzing and predicting one-dimensional and multidimensional time series. On the basis of the T-test, the effectiveness of the recurrent R-SSA forecast of the average annual height of snow and the number of days with snow cover is shown.

For all the meteorological quantities considered, the periodicity of their changes, the standard deviation, the maximum deviation and the relative error were obtained. As a result of the selection of the main components $(1,3$, and 13), prognostic trends of changes in the studied variables were obtained, periods of their increase and decrease were revealed, and predicted values of the average decade height of snow cover and the number of days for the period 2018-2022 were obtained

As a result of the use of the method of singular-spectral analysis, the forecast of such snow cover characteristics of the southern ETR, such as the average decade height of the snow cover and the number of days with snow cover of the southern ETR for 2018-2022, was made. The identified general trends in the studied characteristics of snow cover in the south of the ETR for the period up to 2022 allow characterizing regional climate changes in the south of the European part of Russia as an integral part of contemporary global warming.

snow depth, singular-spectral analysis, prediction, main components, mode residuals, T-test.

\section{Введение}

Изменения режима зимних осадков и снежного покрова (СП) могут считаться комплексным индикатором климата холодного сезона, отражаюшим изменения режима температуры, режима осадков, частоты оттепелей и т.д. Несмотря на значительные потребности в достоверной информации об изменениях характеристик зимних осадков и формирования снежного покрова в различных климатических зонах юга России, влияющего на 
жизнедеятельность и продуктивность природных экосистем и сельскохозяйственных культур, региональные особенности современных климатических изменений и их последствий до настоящего времени недостаточно изучены.

На фоне глобального потепления, наблюдаемого в последние десятилетия, происходят изменения всех характеристик снежного покрова $[1,2,3]$. При потеплении климата ожидается сокращение площади снежного покрова на значительной части территории страны. Кроме того, следует подчеркнуть важность знания закономерностей распределения снежного покрова для оценки климатических ресурсов страны, к которым, несомненно, относится снежный покров. Изменения климата и вызванные этим последствия неоднородны в пространстве и по сезонам, в частности, это касается и основных характеристик снежного покрова: среднедекадной высоты снега, числа дней со СП.

Увеличение продолжительности залегания снежного покрова на европейской территории России сопровождается ростом средней за зимний период высоты снежного покрова. Тенденции роста средней за зимний период высоты снежного покрова наблюдаются на большсй части территории России.

В Климатической доктрине Российской Федерации говорится, что поскольку значительная часть территории Российской Федерации находится в области максимальных (как наблюдаемых, так и прогнозируемых) изменений климата, то надо учитывать особенности Российской Федерации при решении проблемы изменений климата.

\section{Методы и материалы исследований}

Наблюдения за снежным покровом начинаются в осенний период с момента появления первого (временного) снежного покрова и продолжаются до полного его исчезновения в весенний период. Снежный покров наблюдается по установленным стационарно (постоянным) рейкам и с помощью снегосъемок. Число дней со снежным покровом измеряются только по постоянной рейке. Для характеристики среднедекадной высоты снежного покрова вычисляют средние ее значения по декадам месяцев холодного сезона. По отсчетам трех реек ежедневно вычисляется среднее значение снежного покрова. Значение меньшее 0,5 см, записывается как 0 , большее или равное 0,5 см, как 1 см. Для оценки продолжительности залегания снежного покрова используются число дней с покрытием снегом более 50 \% территории вокруг метеостанции.

Объектом исследования в данной статье является прогноз характеристик снежного покрова (среднедекадная высота снежного покрова и число дней со снежным покровом) в холодный период на юге европейской территории России до 2022 года. В основу исследований положены материалы многолетних наблюдений с 1960 по 2017 гг. высоты снежного покрова и дней со снежным покровом по данным м/станций Владикавказ, Изобильный, Каменномостский, Кисловодск, Майкоп, Моздок, Нальчик, Прохладная, Ставро- 
поль, Теберда, Черкесск (данные предоставлены Северо-Кавказским управлением гидрометеослужбы Российской Федерации).

Для получения среднегодового ряда усреднялись среднедекадные высоты за 7 месяцев (октябрь, ноябрь, декабрь, январь, февраль, март, апрель) холодных сезонов 1960/61-2016/17 гг. Был проведен пошаговый анализ и прогноз высоты снежного покрова и числа дней со снежным покровом методом «Гусеница SSA» для 11 м/станщии [4-6]. Результатом применения метода является разложение временного ряда на простые компоненты: медленные тренды, сезонные и другие периодические или колебательные составляющие, а также шумовые компоненты. Полученное разложение может служить основой прогнозирования, как самого ряда, так и его отдельных составляющих.

Техническую основу метода сингулярно-спектрального анализа («Гусеница»-SSA) составляет сингулярное разложение матрицы, столбцами которой являются вектора - отрезки ряда длины $\mathrm{L}$, основного параметра метода, называемого шириной окна. Для того, чтобы выделить какую-то составляющую ряда или отделить сигнал от шума, необходимо найти соответствующие искомой составляющей компоненты разложения, сгруппировать их и восстановлением получить искомый ряд. Метод сингулярно-спектрального анализа (SSA), используемый в работе, позволяет сделать следующее:

- выделить конечное число компонент во временных рядах, которые были рассмотрены в рассматриваемых регулярных интервалах;

- найти скрытые периодичности;

- на основе отобранных компонентов произвести сглаживание начальных данных;

- извлечь компоненты с известным периодом;

- прогнозировать будущие значения в наблюдаемой зависимости $[7,8]$

Качество прогноза в нашей работе проверялось с использованием фактического ряда (1992-2012 гг.) по расчетам следующих критериев:

1) стандартное отклонение и максимальное отклонение прогнозных значений от фактических;

2) Т-тест для получения статистически значимой разницы (или равенства) между средними значениями фактического ряда (1992-2012 гг.) и прогнозного ряда на этот же период;

$3)$ относительная ошибка прогноза $\delta(\%)$

$$
\delta=\frac{1}{n} \sum_{i=1}^{n}\left|\frac{y_{\phi}-y_{\text {пр }}}{y_{\phi}}\right| .100 \%
$$




\section{Результаты исследований и их обсуждение}

Прогноз характеристик снежного покрова юга ЕТР, а также общие тенденции изменения исследуемых характеристик был осуществлен методом сингулярно-спектрального анализа (SSA). Был проведен пошаговый анализ и прогноз высоты снежного покрова и числа дней со снежным покровом методом «Гусеница SSA». Для исследования структуры ряда было проведено преобразование, разложение, группировка и восстановление ряда, для чего использовался вариант метода «Гусеница SSA» без центрирования матрицы наблюдений, соответствующей ряду. Была рассмотрена периодограмма среднедекадной высоты снежного покрова за исследуемый период и выделены наиболее значительные периоды ряда, соответствующие максимальным мощностям. Если временной ряд имеет спектральное разложение вида:

$$
X(t)=M(x)+A_{n} \cos \omega_{n} \tau+B_{n} \sin \omega_{n} \tau
$$

то периодограмма состоит из точек $\left(\omega_{n} ; A_{n}^{2}+B_{n}^{2}\right)$. Величина $P=\left(A_{n}^{2}+B_{n}^{2}\right)-$ это квадрат модуля, характеризующий мощность соответствующей гармоники с периодом $\mathrm{T}$. Большой пик в области некоторой частоты $\omega_{n}$ указывает на то, что в спектральном разложении автокорреляционной функции присутствует соответствующая гармоническая компонента. Чем выше и резче выделен пик, тем большая часть мощности сосредоточена около частоты $\omega_{n}$ и тем большую роль играет эта частота в описании соответствующего случайного процесса или временного ряда. Определены периоды изменения высоты снежного покрова на юге России, соответствующие максимальным пикам мощности: 17 лет, 10 лет, 8 лет, 5 лет.

Для анализа структуры временного ряда методом SSA были подобраны оптимальные параметры - ширина окна и количество главных компонент (ГК). Возьмем ширину окна, равную $\mathrm{L}=20(\mathrm{~L}=52-33+1)$. Далее были определены коэффициенты для линейно-рекуррентной формулы (3), описывающей восстановленный ряд:

$$
\begin{aligned}
& f_{n}=C_{1} 0,996^{n}+C_{2} 1,011^{n} \cos \left(\frac{2 \pi m}{6,47}+\varphi_{2}\right)+C_{3} 0,992^{n} \cos \left(\frac{\pi \pi n}{4,89}+\varphi_{3}+C_{4} 0,983^{n} \cos \left(\frac{2 \pi m}{17,23}+\varphi_{4}\right)+\right. \\
& \left.\left.C_{5} 0,978^{n} \cos \left(\frac{2 \pi n}{2,24}+\varphi_{5}\right)+C_{6} 0,974^{n} \cos \left(\frac{2 \pi n}{3.14}+\varphi_{4}\right)+C_{7} 0,97^{n} \cos 2 \pi n / 3,99\right)+\varphi_{5}\right)
\end{aligned}
$$

После восстановления и аппроксимации ряда линейно-рекуррентной формулой (3) провели прогнозирование ряда (жирная ломаная, рис. 1). Для проверки качества прогноза взяли упреждающий ряд с 1992 г. Используя метод сингулярно-спектрального анализа построили прогнозный ряд с 1992 по 2012 год, и продолжили прогноз на последующие десять точек по 2022 г. Вертикальная черная линия отделяет начало прогноза от фактических данных.

Для подтверждения адекватности модели исходному ряду необходимо доказать, что ряд остатков является случайным и подчиняется нормальному закону распределений. Поэтому следующее предположение состоит в 


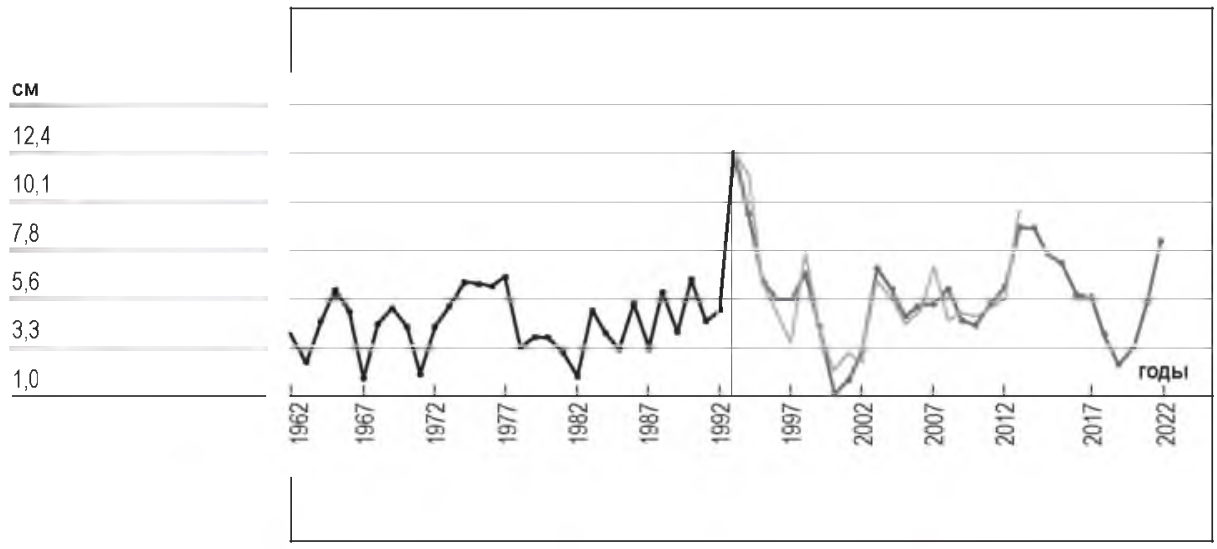

Рис. 1. Рекурентный способ прогноза ряда среднекадной высоты снежного покрова на 1992-2022 гг. ( $L=20$, ГK (1-3))

Fig. 1. Recurrent metod of forecasting a number of average decade height of snow cover for 1992-2022 (L = 20, GK (1-3)).

том, что ряд остатков (ошибок), на основе которого строится прогноз, должен состоять из независимых одинаково распределенных случайных величин. В этом случае мы можем пользоваться выборочной моделью для прогноза. Таким образом, остатки должны быть:
а) некоррелированные.
б) нормально распределены.

На рисунке 2 видно, что автокорреляционная функция в целом подтверждает некоррелированность остатков и сравнение теоретической и эмпирической функций распределения подтверждает нормальность остатков.

Таким образом, можно считать, что структура временного ряда выявлена и восстановлена, поэтому структура ряда была продолжена, то есть был получен прогноз (продолжение) временного ряда. Результаты прогноза представлены в таблице 1 .

\section{Точность прогноза}

1. Стандартное отклонение $1,03 \mathrm{~cm}$;

2. Максимальное отклонение $2,05 \mathrm{cm;}$

3. Относительная ошибка $17,7 \%$;

4. Т-тест (Sig. 0,91>0,05) 5,7 см (исходный) $\approx 5,6$ см (прогнозный). 

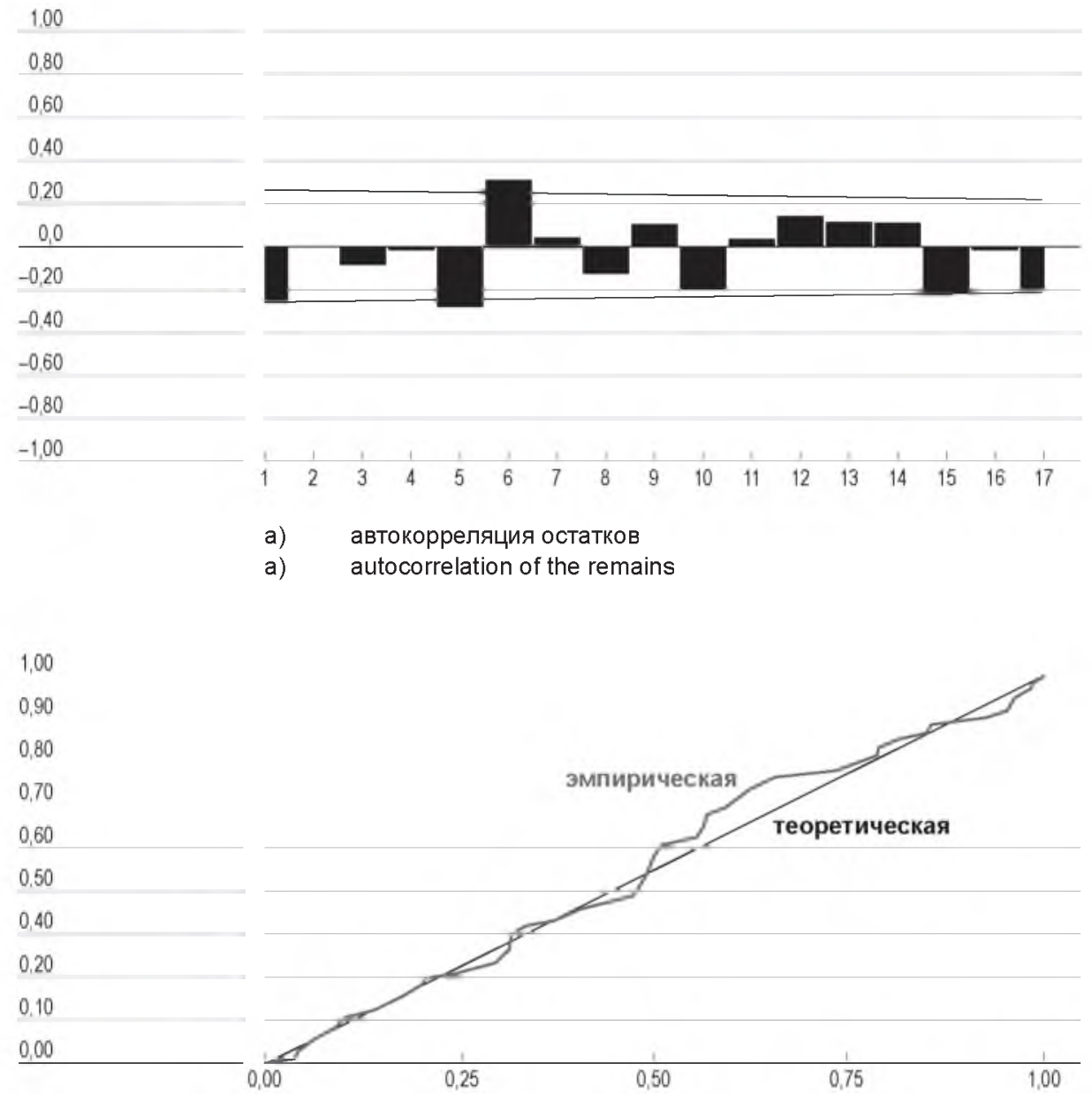

б) нормализованный график остатков восстановленного ряда

b) the normalized scheduleof the remains of the restored row

Pис. 2.

\section{Анализ нормальных остатков.}

Fig. 2. Analysis of normality of the remains.

Минимальное значение высоты снега 2,3 см придется на 2019 год. Далее ожидается увеличение высоты снега до 8,2 см в 2022 году.

При использовании одной главной компоненты получили общую положительную тенденцию тренда (рис. 3) из которой следовало, что увеличения среднедекадной высоты снега с 1992 года продолжает тенденцию, выявленную за период 1961-1991 гг. 
ПРОГНОЗ СРЕДНЕДЕКАДНОЙ ВЫСОТЫ СНЕЖНОГО ПОКРОВА Table 1. Forecast of a decade height of snow cover

\begin{tabular}{l|l|l|l}
\hline $\begin{array}{l}\text { № } \\
\text { nлn }\end{array}$ & Годы & $\begin{array}{l}\text { Исходный } \\
\text { ряд, см }\end{array}$ & $\begin{array}{l}\text { Прогнозный } \\
\text { ряд, см }\end{array}$ \\
\hline 1 & 1961 & 3,8 & - \\
\hline$\cdots$ & $\cdots$ & $\cdots$ & $\cdots$ \\
\hline 32 & 1992 & 12,4 & 12,4 \\
\hline 33 & 1993 & 11,4 & 9,5 \\
\hline 34 & 1994 & 6,3 & 6,3 \\
\hline 35 & 1995 & 4,6 & 5,5 \\
\hline 36 & 1996 & 3,4 & 5,5 \\
\hline 37 & 1997 & 7,8 & 6,6 \\
\hline
\end{tabular}

\begin{tabular}{|l|l|l|l}
\hline $\begin{array}{l}\text { № } \\
\text { n/n }\end{array}$ & Годы & $\begin{array}{l}\text { Исходный } \\
\text { ряд, см }\end{array}$ & $\begin{array}{l}\text { Прогнозный } \\
\text { ряд, см }\end{array}$ \\
\hline 38 & 1998 & 3,9 & 4,2 \\
\hline 39 & 1999 & 2,0 & 1,0 \\
\hline$\ldots$ & $\ldots$ & $\ldots$ & $\ldots$ \\
\hline 58 & 2018 & - & 3,8 \\
\hline 59 & 2019 & - & 2,3 \\
\hline 60 & 2020 & - & 3,1 \\
\hline 61 & 2021 & - & 5,3 \\
\hline 62 & 2022 & - & 8,2 \\
\hline
\end{tabular}

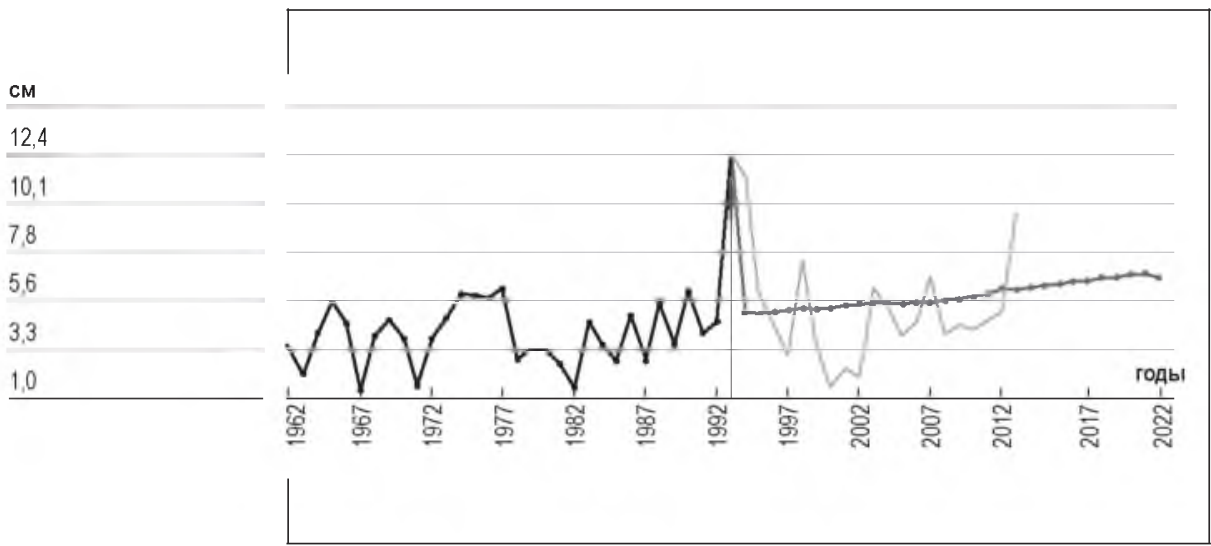

Pnc. 3.

Прогноз среднедекадовой высоты снежного покрова 1992-2022 гг. (ГК1).

Fig. 3. Forecast of average decade height of snow cover, 1992-2022 (GK1)

Были проведены аналогичные исследования по прогнозу числа дней со снежным покровом на юге России (рис. 4). Выявленные периоды в ряду продолжительности залегания снежного покрова составляют 17 лет, 10 лет, 4 и 5 лет.

Точность прогноза

1. Стандартное отклонение 8 дней

2. Максимальное отклонение 15 дней

3. Относительная ошибка $11,6 \%$;

4. Т-тест (Sig. 0,982 > 0,05) 61,3 (исходный) $\approx 61,4$ (прогнозный). 

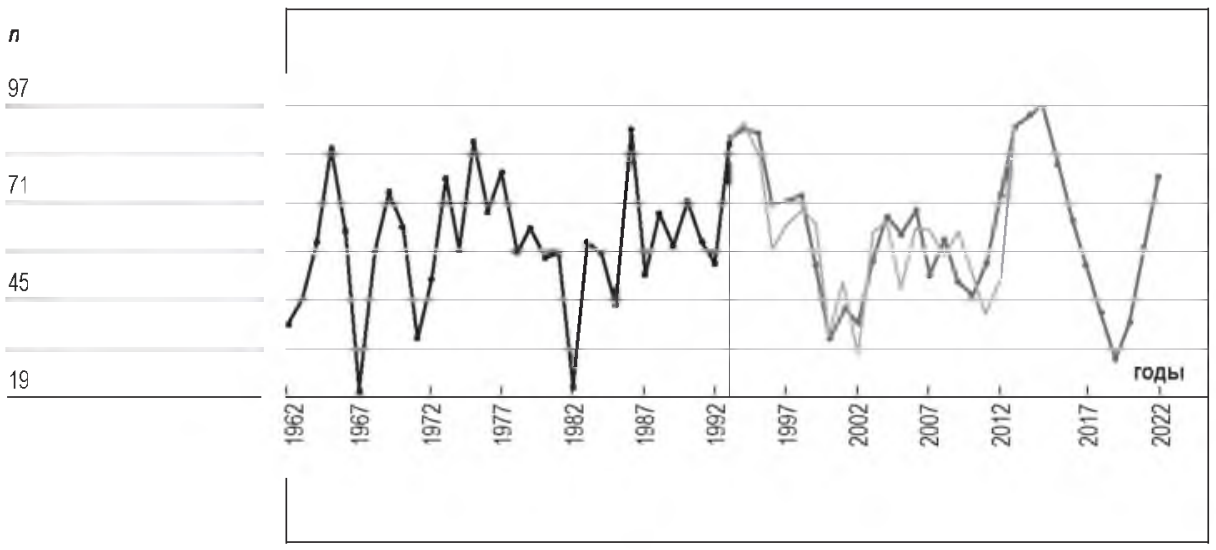

Рис. 4.

Прогноз числа дней со снежным покровом, 1992-2022 гг., $(L=23$, ГK $=10)$.

Fig. 4. Forecast of the number of days with snow cover, 1992-2022, $(L=23, G K=10)$.

Периодичность изменения ряда за исследуемый период: 4 года, 5 лет, 10 лет, 17 лет.

Из рисунка 5 видно, что при ГКІ имеем медленный тренд. характеризующий тенденцию снижения числа дней со снежным покровом и продолжительности залегания снежного покрова на юге ЕТР.

При выбранном количестве главных компонент, равном трем, ГК $=3$, получили прогнозную кривую близкую к синусоиде, демонстрирующую периоды увеличения и снижения тенденции числа дней со снегом. С 2005 года по 2022 год прослеживается период снижения продолжительности залегания снежного покрова на юге ЕТР.

\section{Выводы}

Нелинейные тренды значений высоты снежного покрова и числа дней со снежным покровом, полученные с помощью метода SSA, демонстрируют наличие осцилляций колебаний с общими периодами порядка 17, 10 и 5 лет. Настоящий временной этап (сезоны 2018/19 гг. и 2019/20 гг.) соответствует минимуму в ожидаемых значениях высоты снежного покрова (2,3 см и 3,1 см) и числа дней (36 и 34 дня). В дальнейшем (сезон 2021/22 г.) можно ожидать периода локального похолодания с максимальной высотой снежного покрова $(8,2 \mathrm{~cm})$ и числа дней (66 дня).

Все полученные тенденции прогнозных значений характеристик снежного покрова на 2018-2022 гг. продолжают тенденции изменений характеристик снежного покрова за 1961-2017 гг. и позволяют характеризовать региональные изменения климата юга европейской части России как составную часть современного глобального потепления. 


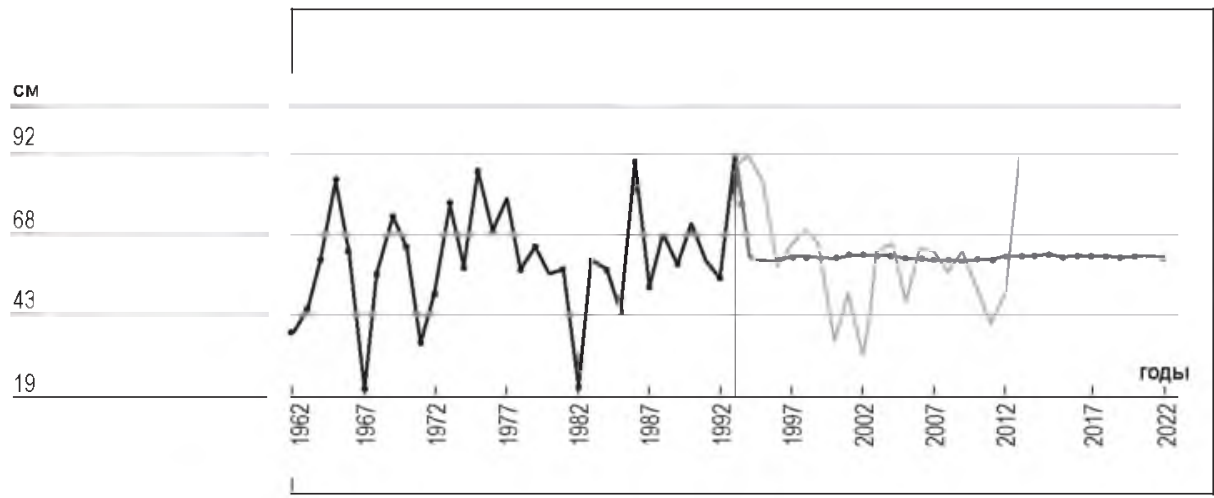

а) восстановленный ряд числа дней со СП по одной главной компоненте (ГК1):

б) restored series of the number of days with snow cover on one main component

$\mathrm{CM}$

92

68

43

19

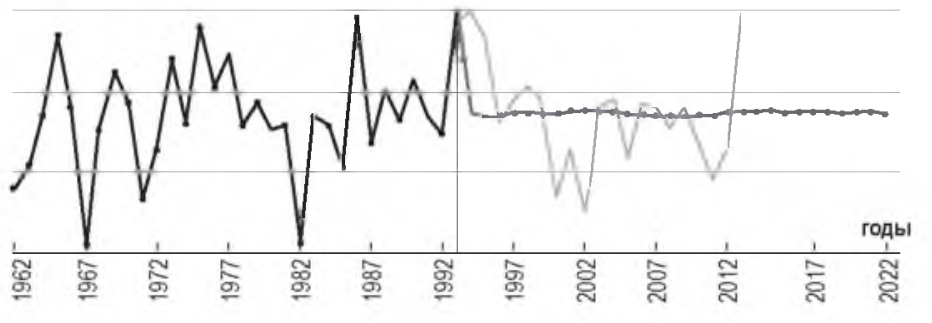

a) восстановленный ряд числа дней со СП по трем главным компонентам (ГК1);

b) restored series of days with snow cover by three main component (GK3)

Рис. 5.

Тенденции изменения числа дней со снежным покровом.

Fig. 5. Trends in the number of days with snow cover

\section{Би6лиографический список}

1. IPCC. Summary for Policy-makers of the Special Report "Managing the Risks of Extreme Events and Disasters to Advance Climate Change Adaptation," Ed. by C. B. Field, V. Barros, T. F. Stocker, et al Special Report of Working Groups I and II of the Intergovernmental Panel on Climate Change (Cambridge, New York, USA, 2012).

2. Roshydromet's Second Assessment Report on Climate Change and its Consequences on the Territory of the Russian Federation (2013) Rosgidromet, Moscow. 1009.

3. 16th Session of the Commission for Climatology (CCl-16), Heidelberg, Germany, 3-8 July 2014. Resource: www.ccl-16.wmo.int 
4. ашилова А.А., Кешева Л.А. Анализ высоты снежного покрова в предгорной части Северного Кавказа // Международная научная конференция по региональным проблемам гидрометеорологии и мониторинга окружающей среды. Казань, 2012. С. 206-207

5. Ташилова А.А., Кешева Л.А., Пшихачева И.Н., Таубекова З.А. Возможности прогнозирования временного климатического ряда с помощью метода «Гусеница» -SSA // Сборник научных трудов Международной научной конференции с элементами научной школы «Инновационные методы и средства исследований в области физики атмосферы, гидрометеорологии, экологии и изменения климата». Ставрополь, 2013. С.86-89.

6. Структурный анализ и прогноз зимних осадков методом «Caterpillar»-SSA / A.A. Ташилова [и др.] // Доклады AMAH. 2013. №1. С. 103-106.

7. Голяндина Н.Э. Метод «Гусеница» -SSA Прогноз временных рядов. СПб: Изд-во СПбГУ, 2004. 52 с.

8. Caterpillar-SSA Copyright (C) 1996-2018 GistaT Group. All rights reserved. Access mode: http://www.gistatgroup.com (date of application: 20.02.2018).

\section{References}

1. IPCC. Summary for Policy-makers of the Special Report "Managing the Risks of Extreme Events and Disasters to Advance Climate Change Adaptation," Ed. by C. B. Field, V. Barros, T. F. Stocker, et al. Special Report of Working Groups I and II of the Intergovernmental Panel on Climate Change (Cambridge, New York, USA, 2012).

2. Roshydromet's Second Assessment Report on Climate Change and its Consequences on the Territory of the Russian Federation. (2013) Roshidromet, Moscow. 1009.

3. 16th Session of the Commission for Climatology (CCl-16), Heidelberg, Germany, 3-8 July 2014. Resource: www.ccl-16.wmo.int.

4. Tashilova A.A., Kesheva L.A. Analiz vysoty snezhnogo pokrova v predgornoy chasti Severnogo Kavkaza (Analysis of snow depth in the foothills of the North Caucasus )//Mezhdunarodnaya nauchnaya konferentsiya po regional'nym problemam gidrometeorologii i monitoringa okruzhayushchey sredy. Kazan', 2012. P. 206-207. (in Russ).

5. Tashilova A.A., Kesheva L.A., Pshikhacheva I.N., Taubekova Z.A. Vozmozhnosti prognozirovaniya vremennogo klimaticheskogo ryada s pomoshch'yu metoda "Gusenitsa»-SSA (The predictive ability of the temporary climatic series using the method of "Caterpillar"-SSA) // Sbornik nauchnykh trudov Mezhdunarodnoy nauchnoy konferentsii s elementami nauchnoy shkoly «Innovatsionnyye metody i sredstva issledovaniy $v$ oblasti fiziki atmosfery, gidrometeorologii, ekologii i izmeneniya klimata». Stavropol', 2013. P. 86-89. (in Russ).

6. Strukturnyy analiz i prognoz zimnikh osadkov metodom «Gusenitsa»-SSA (Structural analysis and prediction of winter precipitation method "Caterpillar"-SSA) / A.A. Tashilova [i dr.] // "Doklady AMAN». 2013. №1. P. 103-106. (in Russ). 
7. Golyandina N.E. Metod "Gusenitsa»-SSA Prognoz vremennykh ryadov. (The method of "Caterpillar"-SSA Forecast time series). SPb Izd-vo SPbGU, 2004. 52 p. (in Russ).

8. Caterpillar-SSA Copyright ( ) 1996-2018 GistaT Group. All rights reserved. Access mode: http://www.gistatgroup.com (date of application: 20.02 .2018 )

\section{O6 авторах}

Ашабоков Борис Азреталиевич, доктор физико-математических наук, профессор, заведующий отделом физики облаков Федерального государственного бюджетного учреждения «Высокогорный геофизический институт» (ФГБУ «ВГИ»), заведующий отделом математических методов исследования сложных систем и процесСОВ ФГБУН «Институт информатики и проблем регионального управления КБНЦ РАН». Scopus ID: 6505916110 Researcher ID: K-4299-2015, Телефон (928) 707-29-52, E-mail: ashabokov. boris@mail.ru.

Ташилова Алла Амарбиевна, кандидат физико-математических наук, доцент, старший научный сотрудник отдела фризики облаков ФГБУ «ВГИ». Scopus ID: 57191577384, Researcher ID: K-4321-2015 Телесон (928) 692-46-29, E-mail: tashilovaa@mail.ru.

Кешева Лара Асировна, кандидат физико-математических наук, старший научный сотрудник отдела фризики облаков ФГБУ «ВГИ» Scopus ID: 57191577471, Researcher ID: K-4261-2015, Телесон (903) 490-47-75, E-mail: kesheva.lara@yandex.ru.

\section{About the authors}

Ashabokov Boris Azretaliyevich, doctor of physical and mathematical sciences professor, head of department of physics of clouds of Federal state budgetary institution "High-Mountain Geophysical Institute", head of department of mathematical methods of a research of difficult systems and processes of FGBUN "Institute of Computer Science and Problems of Regional Management, Kabardino-Balkaria Research Center, Russian Academy of Sciences". Scopus ID: 6505916110 Researcher ID: K-4299-2015, Phone: (928) 707-29-52, E-mail: ashabokov.boris@mail.ru.

Tashilova Alla Amarbiyevna, candidate of physical and mathematical sciences, associate professor, senior research associate of department of physics of clouds of Federal state budgetary institution "High-Mountain Geophysical Institute", Scopus ID: 57191577384 Researcher ID: K-4321-2015, Phone: (928) 692-46-29, E-mail: tashilovaa@ mail. ru

Kesheva Lara Asirovna, candidate of physical and mathematical sciences, senior research associate of department of physics of clouds of Federal state budgetary institution "High-Mountain Geophysical Institute". Scopus ID: 57191577471 Researcher ID: K-4261-2015 Phone: (903) 490-47-75, E-mail: kesheva.lara@yandex.ru. 